Original Research Paper

\title{
Comparative Assessment of Analytical models for the ULS Resistance Verification of Structural Glass Elements under Variable Loads
}

\author{
Chiara Bedon and Claudio Amadio \\ Department of Engineering and Architecture (DIA), University of Trieste, Trieste, Italy
}

Article history

Received: 02-11-2016

Revised: $12-11-2016$

Accepted: 15-03-2017

Corresponding Author:

Chiara Bedon

Department of Engineering and Architecture (DIA), University of Trieste, Trieste, Italy

Email: bedon@dicar.units.it

\begin{abstract}
The design of glass structures, due to the intrinsic material properties, is mainly governed by the typical tensile brittle behavior of the material. In this regard, a currently open question related to the use of glass in buildings as a load-bearing constructional material, is represented by the correct estimation of static fatigue phenomena due to a generic combination of design actions. In this study, taking advantage of past literature contributions and existing design standards for glass, a novel analytical formulation is proposed for the resistance verification of a given structural glass elements under a Ultimate Limit State (ULS) combination of variable loads. The novel proposal is assessed towards three existing analytical formulations, based on two worked examples as well as an extended analytical analysis. In conclusion, the potential and criticisms of the examined approaches are discussed.
\end{abstract}

Keywords: Structural Glass, Laminated Glass, Design Strength, Ultimate Limit State (ULS) Variable Loads, Resistance Verification, Analytical Models

\section{Introduction and State-of-the-Art}

Glass intended as a load-bearing construction material for buildings, as known, is typically characterized by limited tensile resistance and brittle behavior. The resistance verification of structural glass elements, as a result, represents an open question for researchers and designers.

On one side, research studies are aimed to provide sufficiently wide safety margins and practical formulations to designers, towards the development and calibration of standardized design rules. On the other hand, professional engineers are typically aimed to minimize a given design problem, i.e., to optimize the weight and cost of load-bearing elements, compared to their structural performance. In doing so, an appropriate balance should be generally found in terms of applicability of a given design method and rule, as well as sensitivity towards possible errors in design or uncertainties on the material.

Major applications of load-bearing glass components can be in fact found in the form of roofs, facades, stairs, columns, etc. (Fig. 1), including a wide range of possible loading and boundary configurations, as well as un- conventional loading conditions like dynamic loads or impacts. Uncertainties in their design can also arise from the combined use of glass together with other bearing components (typically including steel, aluminum, timber of fiber-reinforced polymers), but especially from the material itself (crack propagation, etc.).

As in the case of traditional construction materials, in this regard, several design standards (ASTM E130012ae1, 2002; CAN/CGSB 12.20-M89, 1989; CNR-DT 210/2013, 2013; DIN 18008: 2010-12, 2010; ÖNORM B 3716, 2013; NEN 2608: 2011, 2011; prEN 13474-1: 2007, 2007; prEN 16612: 2013, 2013), guidelines and recommendations (Feldmann et al., 2014; Larcher et al., 2016) have been proposed in the last years, aimed to provide appropriate methods and formulations voted to safe design principles.

For this purpose, aiming to provide robust knowledge and/ or experimental background for the implementation of design recommendations, in the last years several researchers explored specific aspects related to the structural performance of glass loadbearing elements, both at the material and assembly level (Maniatis and Elstner, 2016; Hagl, 2016; Badalassi et al., 2014; Ballarini et al., 2016; etc.), 
with careful consideration for their resistance and deformability verification, including buckling phenomena (Bedon and Amadio, 2015; 2016). Most of these studies were related to the implementation of appropriate formulations able to account for the cumulative stress effects in glass elements (i.e., static fatigue effects) subjected to a combination of variable loads, see for example (Wiederhorn and Bolz, 1970; Brown, 1972; Beason and Morgan, 1984; Sedlacek et al., 1995; Fischer-Cripps and Collins, 1995; Overend, 2010; Franco and Royer-Carfagni, 2015).

In this study, based on recent literature efforts and existing design standards for structural glass elements, a novel verification approach (WA, in the following), simple in use but rather accurate for the detection of the failure configuration is proposed. Given a generic combination of $N$ design actions with specific magnitude and characteristic duration, the formulation takes advantage of a linear cumulative damage model and a weighted average calculation of an equivalent $k_{\text {mod,w }}$ coefficient well representative of the dominant action (both in terms of time loading and stress level). As a result, under a generic combination of loads, accurate estimations can be obtained for the expected failure configuration, as well as for intermediate levels of damage, compared to existing formulations.

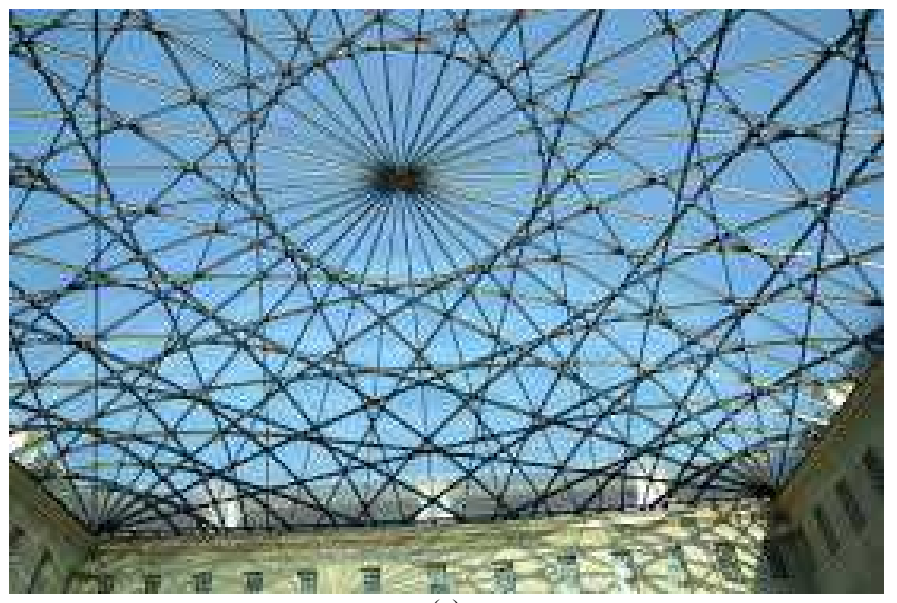

(a)

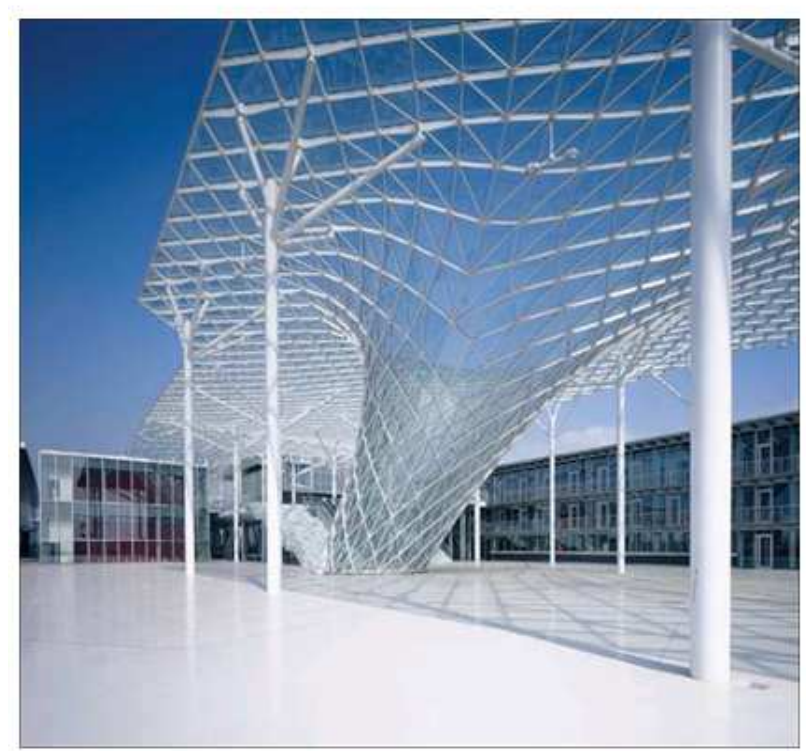

(b)

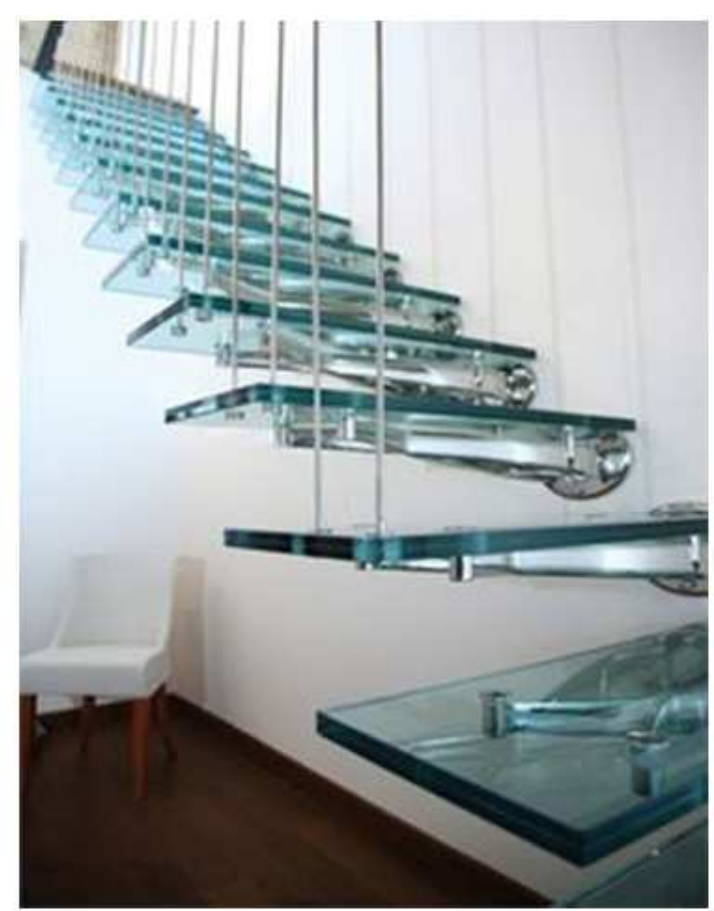

(c)

Fig. 1. Examples of glass structural systems, including (a) a roof; (b) a complex assembly and (c) a stair (www.pinterest.com) 
Three past analytical models for the Ultimate Limit State (ULS) resistance verification of glass elements are in fact taken into account, in order to establish-based on two worked examples of practical interest as well as on an extended analytical study the accuracy and possible criticisms of the novel WA proposal. The approximate linear formulation implemented in the European standard provisions (prEN 16612: 2013, 2013, labelled as 'prEN', in the following) and the Palmgren-Miner based linear method in use in the Italian code CNR-DT 210/2013 for structural glass (CNR-DT 210/2013, 2013; labelled as 'CNR', in the following) are first considered.

Further analytical comparisons are then carried out by means of an accurate and robust, non-linear theoretical model presented by Franco and Royer-Carfagni (2015), labelled as ' $F \& R$ ', in the following. The advantage of the latter approach, compared to the existing linear methods, is its capacity to correctly estimate the static fatigue effects in glass due to a generic combination of variable loads with assigned characteristic time loading and magnitude, spanning over the total lifetime of the structural element to verify. For this reason, the F\&R is set as a reference analytical model for the comparative calculations.

As shown in the paper, in fact, both the pr-EN and CNR methods are implemented on a linear damage model and hence simple to use in practical calculations, but the same approaches are roughly approximate and can also provide unsafe predictions, for a given glass element. The novel WA proposal, in this regard, takes advantage of the simplicity of linear formulation, but provides damage estimations that are in rather close correlation with the exponential F\&R predictions, hence representing a valid alternative to the non-linear formulation.

\section{Traditional ULS Design Approach}

Given a structural member in general under a distribution of design actions, according to the Eurocode design approach (EN 1990: 2002, 2002), its resistance verification should be traditionally carried out at the Ultimate Limit State (ULS) by taking into account a combination of loads in the type of:

$$
F_{d, U L S}=\gamma_{G} G+\gamma_{Q} Q_{k, 1}+\gamma_{Q} \sum_{i} \Psi_{0, i} Q_{k, i}
$$

where, $G$ is representative of permanent loads, while $Q_{k, 1}$ and $Q_{k, i}$ denote respectively the dominant and $i$-th variable actions; $\gamma_{G}=1.35$ and $\gamma_{Q}=1.5$ are partial factors and $\Psi_{0, i}$ is the combination factor, to be calculated depending on the assigned variable actions (comprised in the range between 0.7 and 0 , depending on the involved design actions, see (EN 1990: 2002, 2002)).

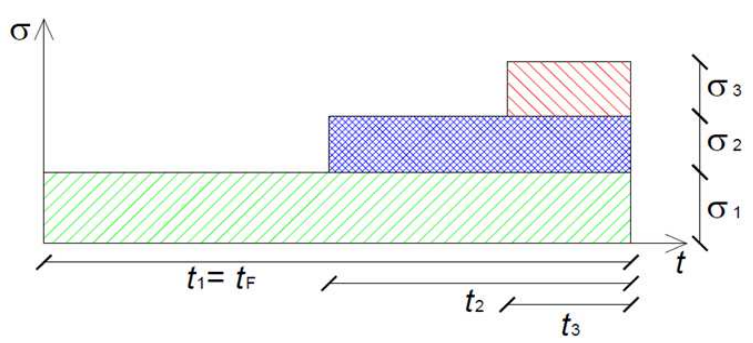

Fig. 2. Stress effects deriving from $j=3$ concurrent design actions with different characteristic duration $t_{j}$ and spanning over the design life-time $t_{F}$

The conventional verification would require then the satisfaction of the condition:

$\sigma_{\max } \leq f_{g d}$

with $\sigma_{\max }$ the ULS maximum stress due to the worst combination of design loads and $f_{g d}$ the material design strength. Differing from other construction materials, for a structural glass element with overall life-time $t_{F}$ and subjected to $j=1, . ., N$ design actions with characteristic duration $t_{j}$ and magnitude (Fig. 2), two main problems implicitly related to the material behavior should be properly solved.

On one side, the first design issue arises in fact from the estimation of the design strength of glass $f_{g d}$, with respect to the assigned ULS combination of $N$ design actions.

At the same time, the maximum stress effects $\sigma_{\max }$ and the related level of damage $D$ deriving from the combined $N$ actions should be correctly calculated, due to the well-known brittle nature of glass. Both the topics are briefly discussed in the following sections.

\section{Design Strength of Glass}

A conventionally accepted expression for the calculation of $f_{g d}$ takes the form:

$$
\begin{aligned}
f_{g d} & =f_{g d, b}+f_{g d, p} \\
& =\frac{k_{\bmod } k_{s p} f_{g k}}{\gamma_{M A}}+\frac{k_{v}\left(f_{b k}-f_{g k}\right)}{\gamma_{M v}}
\end{aligned}
$$

and is given by the sum of two main contributions representative of the tensile resistance of annealed float glass only $\left(f_{g d, b}\right)$ and of possible pre-stressing treatments $\left(f_{g d, p}\right)$, respectively. In Equation 3, $\gamma_{M A}$ and $\gamma_{M v}$ are partial safety factors for material and pre-stressing process, equal to 1.8 and 1.2 .

The second term of Equation 3, moreover, vanishes as far no pre-stressing is applied to glass. Disregarding the $k_{v}$ coefficient representative of the thermal process properties, for a pre-stressed glass element, $f_{g d, p}$ 
basically represent a constant value, due to the available toughening techniques. In it, $f_{g k}$ and $f_{b k}$ denote in fact the characteristic bending strength of annealed (AN, with nominal value of $45 \mathrm{MPa}$ ) and pre-stressed glass (with nominal values in the order of $70 \mathrm{MPa}$ for Heat-Strengthened (HS) and $120 \mathrm{MPa}$ for FullyTempered (FT) glass).

In this context, is it thus clear from Equation 3 that major variations in the design strength $f_{g d}$ are expected on the side of $f_{g d, b}$, where $k_{s p}$ is a coefficient accounting for the surface finishing, while a key role is assigned to the well-known $k_{\text {mod }}$ load duration factor, being this coefficient able to take into account the material strength degradation due to static fatigue phenomena. Assuming $t$ (expressed in hours) as the characteristic duration of a given design action, a practical expression for the estimation of $k_{\text {mod }}$ is given by:

$$
k_{\text {mod }}=0.585 \cdot t^{-1 / 16}
$$

According to Equation 4, the typical values of $k_{\text {mod }}$ for ordinary design actions in constructions lie in a range comprised between 0.26 (permanent loads, 50 years), 0.36 (mid-term loads, 3 months) and 0.88-0.91 (instantaneous loads, 3-5 sec).

\section{Combination of Maximum Stress Effects}

Once the material design strength is defined, i.e., Equation 3, the maximum effects deriving from the $N$ combined actions should be properly estimated, aiming to a standardized ULS resistance verification according with the general form of Equation 2.

Several approaches can be found in literature for the combination of multiple stress states in structural glass elements, including both simplified linear approaches, as well as theoretically robust, non-linear analytical formulations. In this study, taking advantage of recent literature contributions and standard provisions currently in use, an alternative formulation is also proposed for the ULS resistance verification of structural glass elements under a general combination of $N$ design actions and validated towards three selected past formulations.

\section{Existing Analytical Models}

The first reference approach considered in this study is currently implemented in the pr-EN 16612: 2013 provisions for structural glass.

According to Fig. 2 and Equation 1, given a glass member with life-time $t_{F}$ under a ULS combination of $N$ design actions with assigned time loading $t_{j}$ (i.e., $k_{\text {mod, } j}$ the load duration coefficient) and magnitude, the pr-EN formulation estimates the total stress effects $\sigma_{\max }$ as the sum of tensile stresses $\sigma_{j}$ due to the $j$-th action. In the worst ULS combination, the resistance verification is then satisfied as far as the condition:

$$
\frac{\sum_{j=1}^{N} \sigma_{j}}{\max \left(f_{g d, j}\right)} \leq 1
$$

is true.

Despite the simplicity of the formulation, its strong approximation lies in the calculation of strength $f_{g d}$.

In Equation 5, disregarding the number and magnitude of the imposed $N$ design, a single design strength $f_{g d}$ for glass is in fact taken into account. The reference value, according to Equation 3, is calculated for the $j$-th design action associated to the minimum $t_{j}$ (that is, the maximum $k_{\text {mod, } j}$ coefficient). The same verification approach is proposed for AN glass elements as well as for pre-stressed (HS, FT) glass elements.

A different methodology is considered by the CNR formulation implemented in the Italian code for the design of structural glass elements, CNR-DT 210/2013. A Palmgren-Miner based, linear cumulative damage approach is in fact taken into account, differing from the pr-EN standard. As a result, the condition representative of possible failure for a given AN, HS or FT glass element under a ULS combination of $N$ actions is given by:

$\sum_{j=1}^{N} \frac{\sigma_{j}}{f_{g d, j}} \leq 1$

that is by the sum, for each of the imposed $j$-th actions, of the $\sigma_{j}$ stress effect divided by the corresponding design strength $f_{g d, j}$. The latter values must be separately calculated, for each one of the $N$ actions, by means of Equation 3, that is as a function of $N$ load duration coefficients $k_{\text {mod, }}$ (Equation 4).

In order to overcome the intrinsic approximations of the pr-EN and CNR formulations, a theoretically robust, non-linear analytical model has been recently proposed by Franco and Royer-Carfagni (2015), here labelled as 'F\&R'. This subcritical cracks growthbased model takes advantage from a static fatigue consolidated model and consists in an exponential analytical expression for the safety domain of structural glass elements under a generic ULS combination of $N$ design actions. Two key aspects are introduced with the F\&R formulation, compared to the pr-EN and CNR methods.

Any possible pre-stressing effect (i.e., the second term $f_{g d, p}$ of Equation 3 ) is in fact accounted on the side of the actions, rather on the side of the material design strength. The non-linear propagation of damage $D$ in glass, moreover, is correctly considered by properly estimating the cumulative stress effects $\sigma_{j}$ of $N$ actions spanning over different time intervals $t_{j}$. The final expression for the resistance verification is in fact given by: 


$$
\sum_{j=1}^{N} \frac{\left[\left\langle\left(\sum_{i=1}^{j} \sigma_{i}\right)-\sigma_{p}\right\rangle^{+}\right]^{n}-\left[\left\langle\left(\sum_{i=1}^{j-1} \sigma_{i}\right)-\sigma_{p}\right\rangle^{+}\right]^{n}}{\left(f_{g d, b}\right)_{i}^{n}} \leq 1
$$

where at a generic time instant $0 \leq t \leq t_{F}$, the positive part of the resisting domain function $F(t)$ is only considered, i.e.:

$$
\langle F(t)\rangle^{+}=\max \{F(t), 0\}
$$

In Equation 7, $\sigma_{i}$ represents the tensile stress due to each one of the assigned $N$ actions (with $n=16$ ), while $\left(f_{g d, b}\right)_{i}$ denotes the corresponding design resistance, inclusive of the AN glass contribution only (i.e., first term of Equation 3). Finally, $\sigma_{p}=f_{g d, p}$ (i.e., second term of Equation 3 ) is representative of the compressive stress term due to possible toughening processes in HS or FT glass elements.

\section{Weighted Average Analytical Model}

An alternative, Weighted Approach (WA) based on a linear cumulative formulation, is proposed in this study. The WA approach is typically characterized by the simplicity and intuitivism of linear formulations (i.e., prEN or CNR methods).

Two main aspects are implemented in the WA method, however, in order to overcome their implicit approximations.

First, a key role is assigned to the weighted average calculation of an equivalent $k_{m o d, w}$ coefficient accounting both of the characteristic duration $t_{j}$ and stress ratio $R \sigma_{j}$ (i.e., stress vs. design strength ratio) of each $j$-th action. As a result, the estimation errors due to the pr-EN and CNR basic assumptions are expected to be minimized. Moreover, the WA formulation allows to take into account the presence of possible pre-stressing effects on the side of actions rather than on the side of the material design strength, as also in accordance with the exact $F \& R$ formulation.

From a practical point of view, Fig. 2 and 3, the $k_{m o d, w}$ coefficient for AN glass is in fact defined as:

$k_{\mathrm{mod}, w}=\frac{\sum_{j=1}^{N} \sigma_{j} k_{\mathrm{mod}, j}}{\sum_{j=1}^{N} \sigma_{j}}$

As far as any pre-stressing treatment is applied to HS or FT glass elements, Fig. 3, the additional compressive state $\sigma_{p}$ must be also taken into account. As a result, $k_{\text {mod,w }}$ is given by:

$k_{\mathrm{mod}, w}=\frac{\sum_{j=1}^{N} \sigma_{j}^{\prime} k_{\mathrm{mod}, j}}{\sum_{j=1}^{N} \sigma_{j}^{\prime}}$

with:

$\sigma_{j}^{\prime}=\left(\sum_{i=1}^{j} \sigma_{i}\right)-\max \left(\left(\sum_{i=1}^{j-1} \sigma_{i}\right), \sigma_{p}\right) \geq 0$

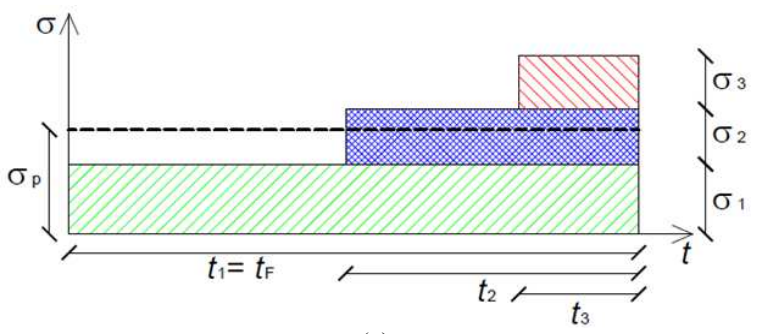

(a)

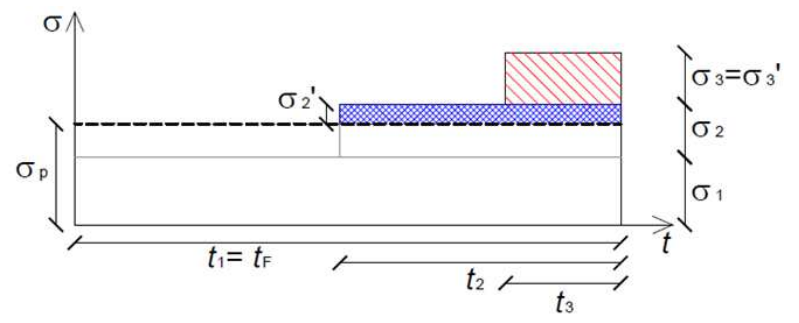

(b)

Fig. 3. Stress effects deriving from $j=3$ concurrent design actions with different characteristic duration $t_{j}$ and spanning over the design life-time $t_{F}$ of a pre-stressed glass element $\left(\sigma_{p} \neq 0\right)$. Charts with evidence of (a) separate stress effects $\sigma_{j}$ and (b) tensile stress increments $\sigma_{j}^{\prime}$ over each time interval.-time $t_{F}$ 
Once $k_{m o d, w}$ is known, the resistance verification of a AN, HS or FT glass panel under a given ULS combination of $\mathrm{N}$ actions can be rationally carried out by satisfying the following condition:

$$
\frac{\left(\sum_{j=1}^{N} \sigma_{j}\right)-\sigma_{p}}{f_{g d, b}^{*}} \leq 1
$$

where, $f_{g d, b}{ }^{*}$ is still given by the first term of Equation 3, with $k_{\text {mod }}=k_{\text {mod,w }}$ (Equation 9). As far as the prestressing effects are included within the design strength (as in the case of the pr-EN and CNR methods), the ULS resistance verification would be governed by the following condition:

$$
\frac{\sum_{j=1}^{N} \sigma_{j}}{f_{g d}^{*}} \leq 1
$$

where, $f_{g d}{ }^{*}$ is given by the sum of both the terms of Equation 3, with $k_{\text {mod }}=k_{\text {mod,w }}$ (Equation 9).

\section{Worked Examples}

In order to check the validity of the novel WA proposal, as well as to establish a general relationship between the pr-EN, CNR and F\&R approaches earlier described, i.e., quantifying the level of approximation of the linear methods compared to the exact F\&R solution, two representative worked examples are critically discussed.

For consistency and simplicity of calculations, the worked examples are referred to a AN laminated glass panel as well as to a pre-stressed (FT) element. In accordance with Fig. 4, all the analytical calculations are then carried out by means of the equivalent thickness formulation proposed in (Galuppi and Royer-Carfagni, 2012).

\section{Case Study 1: Annealed Glass}

The first worked example consists in an annealed (AN) laminated glass panel belonging to a roof subjected to permanent and variable loads.

The reference pane has overall dimensions $b=800$ $\mathrm{mm} \times L=655 \mathrm{~mm}$, is simply supported on the longest edges only and subjected to the combined action of self-weight $G$, snow load $Q_{s}\left(0.8 \mathrm{kN} / \mathrm{m}^{2}\right)$ and maintenance load $Q_{m}\left(0.5 \mathrm{kN} / \mathrm{m}^{2}\right)$. The resisting crosssection is obtained by coupling two glass panes, $h=4$ $\mathrm{mm}$ in thickness and a PVB layer, $h_{\text {int }}=1.52 \mathrm{~mm}$ thick, Fig. 4.

The secant stiffness modulus $G_{\text {int }}$ of the PVB foil, as required by the equivalent thickness calculations, is hence estimated by taking into account the reference time loading $t$ and temperature $T$ for each design action, as well as some PVB mechanical properties derived from literature, Table 1.

\begin{tabular}{|c|c|c|c|c|}
\hline & \multicolumn{3}{|c|}{ Design action } \\
\hline & & $G$ & $Q_{s}$ & $Q_{m}$ \\
\hline$t$ & - & 50 years & 3 months & $3 \mathrm{sec}$ \\
\hline$T$ & {$\left[{ }^{\circ} \mathrm{C}\right]$} & 50 & 30 & 30 \\
\hline$k_{\text {mod }}$ & - & 0.26 & 0.36 & 0.91 \\
\hline$G_{i n t}$ & {$[\mathrm{MPa}]$} & 0.052 & 0.57 & 0.85 \\
\hline$f_{g d, b}$ & {$[\mathrm{MPa}]$} & 6.75 & 9.43 & 20.50 \\
\hline
\end{tabular}

Table 1. Input parameters for the case study 1

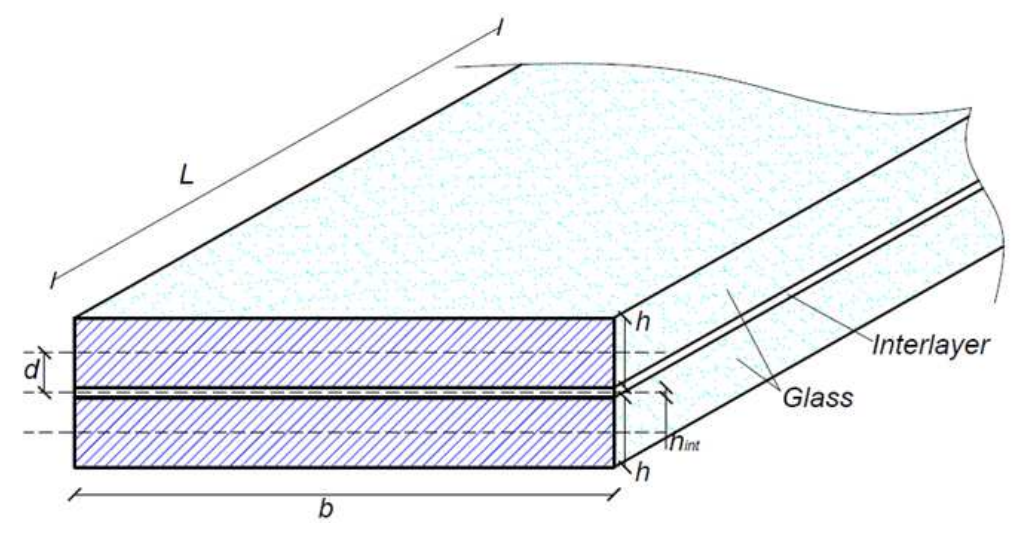

Fig. 4. Reference geometrical configuration for a symmetrical laminated glass panel 
Based on the worst ULS combination of design loads given by Equation 1, assuming the AN laminated glass panel simply supported along two edges only and subjected to uniform pressure, the maximum expected tensile stress due to each $j$-th action is given by:

$\sigma_{\max , j}=0.75 \frac{L^{2}}{\hat{h}_{\sigma}^{2}} F_{d, U L S, j}$

where, $\hat{h}_{\sigma}$ is the equivalent thickness for stresses as provided by (Galuppi and Royer-Carfagni, 2012).

The assigned glass panel is hence verified by following the pr-EN and CNR provisions, as well as on the base of the exact F\&R approach. Finally, calculations are carried out on the base of the novel WA formulation.

\section{pr-EN Method}

Besides the different characteristic duration and reference temperature of the assigned design loads (Table 1), a single value of glass strength $f_{g d}$ is taken into account for the worst ULS combination, that is the $f_{g d}$ design strength given by the shortest design action.

In accordance with Table $1, f_{g d}$ is calculated by means of Equation 3 as a function of the load duration coefficient $k_{\text {mod }}=0.91$ representative of the maintenance load $Q_{m}$. Due to lack of any pre-stressing treatment, Equation 3 reduces then to the first term only, that is:

$$
\begin{aligned}
& f_{g d}=f_{g d, b} \\
& =\frac{k_{\mathrm{mod}} k_{s p} f_{g k}}{\gamma_{M A}}=\frac{0.91 \cdot 1 \cdot 45}{1.8}=22.75 \mathrm{MPa}
\end{aligned}
$$

Given the design value of each $j$-th action and according to the maximum stresses provided by Equation 12, Table 2 and 3, following Equation 4 the ULS resistance verification requires that:

$$
\begin{aligned}
& \frac{\sigma_{1}+\sigma_{2}+\sigma_{3}}{\max \left(f_{g d, 1}, f_{g d, 2}, f_{g d, 3}\right)} \\
& =\frac{2.82+4.33+4.92}{22.75}=0.53 \leq 1
\end{aligned}
$$

As far the left term of Equation 14 is intended as the expected level of damage $D$ for the laminated glass panel object of design-given the input parameters of the worked example it can be seen that the AN laminated panel would be largely adequate to resist the assigned design actions, i.e., being $D=0.53$.

\section{CNR Method}

In the case of the Palmgren-Miner based approach implemented in the CNR Italian document, following Equation 6 and according to the input data collected in Table 2 and 3, the ULS resistance verification of the AN roof panel takes the form:

$$
\begin{aligned}
& \frac{\sigma_{1}}{f_{g d, 1}}+\frac{\sigma_{2}}{f_{g d, 2}}+\frac{\sigma_{3}}{f_{g d, 3}} \\
& =\frac{2.82}{6.50}+\frac{4.33}{9.00}+\frac{4.92}{22.75} \\
& =0.433+0.481+0.216 \\
& =1.13>1
\end{aligned}
$$

\begin{tabular}{|c|c|c|c|c|}
\hline \multirow{2}{*}{$\begin{array}{l}\text { Reference load } \\
\text { (characteristic value) } \\
{\left[\mathrm{kN} / \mathrm{m}^{2}\right]}\end{array}$} & & \multicolumn{3}{|c|}{ Design strength (Equation 3) } \\
\hline & & $\begin{array}{l}f_{g d} \\
{[\mathrm{MPa}]}\end{array}$ & $\begin{array}{l}f_{g d, b} \\
{[\mathrm{MPa}]}\end{array}$ & $\begin{array}{l}f_{g d, p}=\sigma_{p} \\
{[\mathrm{MPa}]}\end{array}$ \\
\hline$G$ & 0.22 & 6.50 & 6.50 & 0 \\
\hline$Q_{s}$ & 0.80 & 9.00 & 9.00 & 0 \\
\hline$Q_{m}$ & 0.50 & 22.75 & 22.75 & 0 \\
\hline
\end{tabular}

Based on Equation 15, consequently, the AN panel would not be able to satisfy the resistance verification, being $D>1$.

Table 2. Reference input data for the ULS resistance verification of the assigned AN roof panel. Maximum stresses

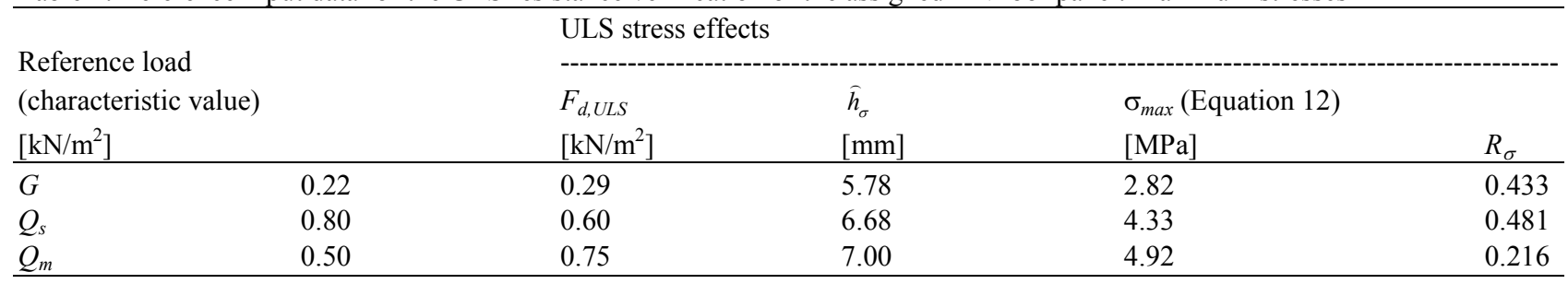

Table 3. Reference input data for the ULS resistance verification of the assigned AN roof panel. Design strength 


\section{F\&R Method}

When the resistance verification of the same roof panel is carried out based on the exact exponential F\&R formulation, the following condition should be satisfied:

$$
\begin{aligned}
& \frac{\left[\left\langle\sigma_{1}-\sigma_{p}\right\rangle^{+}\right]^{n}}{f_{g d, b, 1}^{n}}+\frac{\left[\left\langle\sigma_{1}+\sigma_{2}-\sigma_{p}\right\rangle^{+}\right]^{n}-\left[\left\langle\sigma_{1}-\sigma_{p}\right\rangle^{+}\right]^{n}}{f_{g d, b, 2}^{n}} \\
& +\frac{\left[\left\langle\sigma_{1}+\sigma_{2}+\sigma_{3}-\sigma_{p}\right\rangle^{+}\right]^{n}-\left[\left\langle\sigma_{1}+\sigma_{2}-\sigma_{p}\right\rangle^{+}\right]^{n}}{f_{g d, b, 3}^{n}} \leq 1
\end{aligned}
$$

that is, according to Table 2 and 3 (with $\sigma_{p}=0$ and $n$ $=16)$ :

$$
\begin{aligned}
& \frac{2.82^{16}}{6.50^{16}}+\frac{(2.82+4.33)^{16}-(2.82)^{16}}{9.00^{16}} \\
& +\frac{(2.82+4.33+4.92)^{16}-(2.82+4.33)^{16}}{22.75^{16}}=0.024 \leq 1
\end{aligned}
$$

From the above calculations, it can be noticed that the assigned input data and thickness $h$ for the AN glass panes are such that the resistance verification is largely verified against the assigned ULS combination of loads. A point to be highlighted, in this regard, is the relatively low $D$ value, i.e., being very close to zero despite the moderate stress ratio $R_{\sigma}$ deriving from each one of the assigned $N$ design actions (Table 2).

\section{WA Method}

A rational resistance verification can be based on the novel WA approach proposed in this study. As far as the equivalent $k_{\text {mod,w }}$ coefficient is in fact calculated by means of Equation 9a:

$$
\begin{aligned}
k_{\text {mod, }, w} & =\frac{\sigma_{1} k_{\text {mod, } 1}+\sigma_{2} k_{\text {mod, },}+\sigma_{3} k_{\text {mod, }, 3}}{\sigma_{1}+\sigma_{2}+\sigma_{3}} \\
& =\frac{2.82 \cdot 0.26+4.33 \cdot 0.36+4.92 \cdot 0.91}{2.82+4.33+4.92} \\
& =\frac{0.76+1.56+4.48}{11.63}=\frac{6.77}{11.63}=0.56
\end{aligned}
$$

Some differences from the previously investigated methods can be clearly observed.

The resistance verification requires in fact, according to Equation $11 \mathrm{a}$, that the overall stress effect due to the assigned actions would not exceed the design strength of AN glass calculated as a function of $k_{\text {mod, }}$, that is (with $k_{s p}=1$ ):

$$
f_{g d, b}^{*}=\frac{k_{\mathrm{mod}, w} f_{g k}}{\gamma_{M A}}=\frac{0.56 \cdot 45}{1.8}=14.03 \mathrm{MPa}
$$

The ULS resistance verification is hence satisfied, since from Equation 11a:

$$
\begin{aligned}
& \frac{\sigma_{1}+\sigma_{2}+\sigma_{3}}{f_{g d, b}^{*}} \\
& =\frac{2.82+4.33+4.92}{14.03}=\frac{12.06}{14.03}=0.86 \leq 1
\end{aligned}
$$

Based on Equation 20, it can be seen that the structural capacity of the assigned AN laminated panel would be exploited up to $\approx 90 \%$ its nominal ULS resistance. A certain safety margin is consequently still perceived for its design.

According to linear formulations, a direct correlation is in fact typically observed between the expected level of damage $D$ (i.e., the left term of Equation 5, 6 and 11) and the actual stress ratio for the structural element to verify. This is not the case of Equation 7, due to the exponential increase of $D$.

\section{Discussion of Results}

Based on the collected verification results, as obtained according to the pr-EN, CNR, F\&R and WA approaches, some further iterative calculations were carried out for the same AN glass panel.

In doing so, all the input parameters were kept fix as previously discussed. The assigned glass thickness $h$, on the other hand, was progressively modified and the corresponding level of damage $D$ was calculated following each one of the examined analytical methods, that is on the base of Equation 5-7 and 11.

The so collected comparative results are proposed in Fig. 5. In the figure, it is interesting to notice how the prEN and the CNR formulations are highly approximate the first on the unsafe and the second on the safe sidecompared to the exact F\&R model. From the same figure, however, two additional important aspects can be further observed. It is in fact clear that the high non-linearity of the exact $F \& R$ formulation could negatively affect the design calculations, compared to a linear cumulative approach. As far as small variations in the glass thickness $\mathrm{h}$ are considered (in the order of the decimal part of a millimeter, in this worked example), the corresponding level of damage $D$ exponentially increases. The high sensitivity of other geometrical and/or mechanical input data, in this sense, could also result in improper and hazardous assumptions from inexperienced designers.

The WA formulation, in this sense, can rationally predict the failure configuration (i.e., $D=1$ ) for the assigned AN roof panel, compared to the F\&R approach. The margin of error from the exact solution was found to lie in the order of $\approx 10 \%$. Based on the WA linear cumulative approach for the combination of the stress effects due to multiple design actions, moreover, a certain safety margin is also provided. 
Table 4. Reference input data for the ULS resistance verification of the assigned FT roof panel. Maximum stresses

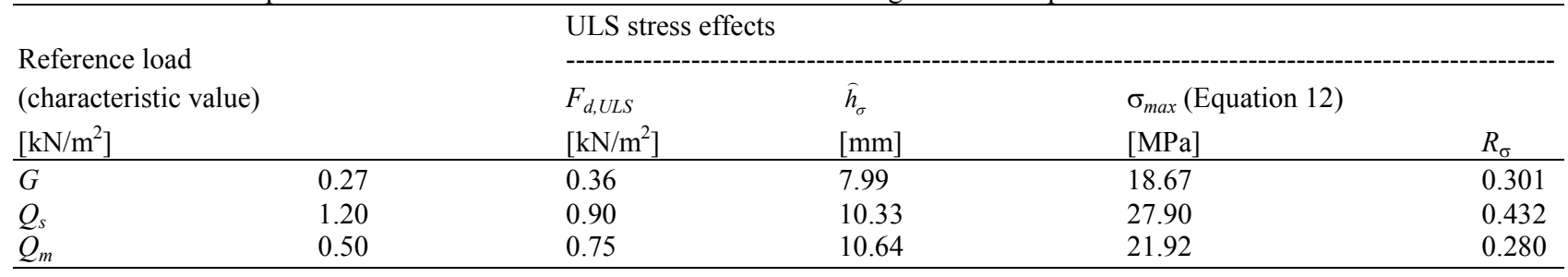

Table 5. Reference input data for the ULS resistance verification of the assigned FT roof panel. Design strength

\begin{tabular}{|c|c|c|c|c|}
\hline \multirow{2}{*}{$\begin{array}{l}\text { Reference load } \\
\text { (characteristic value) } \\
{\left[\mathrm{kN} / \mathrm{m}^{2}\right]}\end{array}$} & & \multicolumn{3}{|c|}{ Design strength (Equation 3) } \\
\hline & & $\begin{array}{l}f_{g d} \\
{[\mathrm{MPa}]}\end{array}$ & $\begin{array}{l}f_{g d, b} \\
{[\mathrm{MPa}]}\end{array}$ & $\begin{array}{l}f_{g d, p} \\
{[\mathrm{MPa}]}\end{array}$ \\
\hline$G$ & 0.27 & 62.05 & 6.50 & 55.55 \\
\hline$Q_{s}$ & 1.20 & 64.55 & 9.00 & 55.55 \\
\hline$Q_{m}$ & 0.50 & 78.30 & 22.75 & 55.55 \\
\hline
\end{tabular}

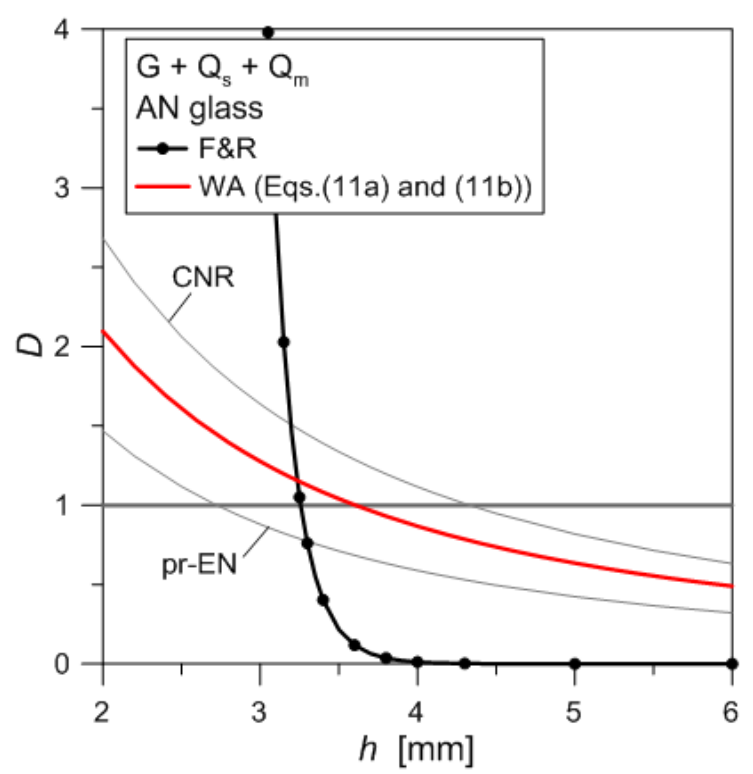

Fig. 5. Comparative analytical calculations for the AN laminated glass roof panel, as a function of the assigned thickness $h$

\section{Case Study 2: Fully-Tempered Glass}

A second case study was also taken into account, in order to draw some further analytical comparisons in the specific case of pre-stressed glass panes.

A roof panel with $b=1000 \mathrm{~mm} \times L=2100 \mathrm{~mm}$ the nominal dimensions was taken into account, in the hypothesis of continuous simply supports along the $b$ long edges only. A laminated glass section composed of two FT glass panes, $h=5 \mathrm{~mm}$ in thickness and a 1.52 $\mathrm{mm}$ PVB foil was then considered.

The ULS verification of the composite section was carried out on the base of the worst combination of selfweight $G$, snow pressure $Q_{s}=1.20 \mathrm{kN} / \mathrm{m}^{2}$ and maintenance load $Q_{m}=0.5 \mathrm{kN} / \mathrm{m}^{2}$, Table 1,4 and 5 . In terms of design resistance, the corresponding value was hence calculated by means of Equation 3, assuming $f_{b k}=$ $120 \mathrm{MPa}$ for FT glass.

\section{pr-EN and CNR Methods}

The pr-EN approach is first applied to the FT roof panel. As in the case of the AN case-study, the resistance verification is hence carried out by comparing the sum of the ULS stress terms $\sigma_{j}$ with the maximum design strength corresponding to the shortest time loading within the $N$ actions, that is:

$$
\begin{aligned}
& \frac{\sigma_{1}+\sigma_{2}+\sigma_{3}}{\max \left(f_{g d, 1}, f_{g d, 2}, f_{g d, 3}\right)}= \\
& =\frac{18.67+27.90+21.92}{78.30}=0.875 \leq 1
\end{aligned}
$$

where, the $\sigma_{j}$ effects are listed in Table 4 and the maximum design strength of glass is still associated to the maintenance load $Q_{m}$ only ( $t=3 \mathrm{sec}$, Table 1 and 5). Differing from the first AN worked example, it can be noticed from Table 5 that the pre-stressing effect gives a meaningful contribution to the resistance of glass. Thanks to the toughening treatment, a rather limited glass thickness can hence sustain the assigned design actions over the total span of $2100 \mathrm{~mm}$. The ULS verification, based on Equation 21, would result satisfied with a moderately high level of expected damage $(D=0.875)$.

As far as the linear cumulative damage model implemented in the CNR document is used, the resistance verification takes the form:

$$
\begin{aligned}
& \frac{\sigma_{1}}{f_{g d, 1}}+\frac{\sigma_{2}}{f_{g d, 2}}+\frac{\sigma_{3}}{f_{g d, 3}} \\
& =\frac{18.67}{62.05}+\frac{27.90}{64.55}+\frac{21.92}{78.30} \\
& =0.301+0.432+0.280=1.013>1
\end{aligned}
$$


and would be consequently not satisfied, being $D>1$.

\section{F\&R Method}

A totally different prediction is found from the exact F\&R formulation, based on the input parameters provided in Table 4 and 5 for the investigated FT panel. The ULS resistance verification is in fact given by:

$$
\begin{aligned}
& \frac{\left(\langle 18.67-55.55\rangle^{+}\right)^{16}}{6.50^{16}} \\
& +\frac{\left(\langle 18.67+27.90-55.55\rangle^{+}\right)^{16}-\left(\langle 18.67-55.55\rangle^{+}\right)^{16}}{9.00^{16}} \\
& +\frac{\left(\langle 18.67+27.90+21.92-55.55\rangle^{+}\right)^{16}}{22.75^{16}} \\
& =\frac{(0)^{16}}{6.50^{16}}+\frac{(0)^{16}-(0)^{16}}{9.00^{16}}+\frac{(12.94)^{16}-(0)^{16}}{22.75^{16}} \\
& =0.00012 \leq 1
\end{aligned}
$$

According to Equation 23, it can be hence observed that a nearly zero level of damage is given by the F\&R method for the examined FT roof panel.

\section{WA Method}

Finally, the novel WA formulation is taken into account. According to Equation 9b, the weighted $k_{m o d, w}$ coefficient needs first to be calculated.

Based on Equation 10 and the input data collected in Table 4 and 5, it can be noticed that the total tensile stress exceeds the imposed pre-compressive state $\sigma_{p}$ as far as all the imposed design actions $G, Q_{s}$ and $Q_{m}$ are concurrent. Following Equation $9 \mathrm{~b}$ and Fig. 3, in particular, the corresponding $k_{m o d, w}$ value is in fact given by:

$$
\begin{aligned}
k_{\mathrm{mod}}^{*}= & \frac{\sigma_{1}^{\prime} k_{\mathrm{mod}, 1}+\sigma_{2}^{\prime} k_{\mathrm{mod}, 2}+\sigma_{3}^{\prime} k_{\mathrm{mod}, 3}}{\sigma_{1}^{\prime}+\sigma_{2}^{\prime}+\sigma_{3}^{\prime}} \\
& =\frac{0+0+12.94 \cdot 0.91}{12.94}=0.91
\end{aligned}
$$

and hence is exactly coincident with the maintenance load coefficient $k_{\text {mod }, 3}$ provided in Table 1 .

Following Equation 11a, the $f_{g d, b}{ }^{*}$ strength value is consequently also known, since (with $k_{s p}=1$ ):

$$
\begin{aligned}
& f_{g d, b}^{*}=f_{g d, b, 3} \\
& =\frac{k_{\mathrm{mod}, w} f_{g k}}{\gamma_{M A}}=\frac{0.91 \cdot 45}{1.8}=22.75 \mathrm{MPa}
\end{aligned}
$$

and the final resistance verification takes the form:

$$
\frac{\sigma_{1}+\sigma_{2}+\sigma_{3}-\sigma_{p}}{f_{g d, b}^{*}}=\frac{12.94}{22.75}=0.57 \leq 1
$$

As far as the pre-stressing contribution is accounted on the side of actions, as in the case of the F\&R formulation, it can be seen from Equation 26 that a medium level of damage $D$ is expected for the FT roof panel. It is interesting to point out, in this context, that the pre-compressive term $\sigma_{p}$ can have important effects on the ULS verification condition and on the corresponding magnitude of expected damage $D$ for the same FT panel. According to Equation $11 \mathrm{~b}$, the ULS resistance check would in fact take the form:

$$
\frac{\sigma_{1}+\sigma_{2}+\sigma_{3}}{f_{g d}^{*}}=\frac{68.49}{78.30}=0.875 \leq 1
$$

and would be exactly coincident with the pr-EN approach, being $f_{g d}{ }^{*}$ given by Equation 3 with $k_{m o d}=$ $k_{\text {mod,w }}=k_{\text {mod,3 }}$.

\section{Discussion of Results}

As far as the FT roof panel was verified by means of the pr-EN, CNR, F\&R and WA analytical formulations, some interesting aspects were observed.

The theoretically exact F\&R formulation, in particular, provided an almost null level of damage in glass, with $D=0.00012$ and a ULS resistance verification totally satisfied. This is not the case of the existing linear methods and specifically of the CNR formulation, which resulted in a negative check for the resistance performance of the same FT roof panel (with $D=1.013$ ). An identical result, with positive verification and a moderately high expected damage in glass (i.e., $D=0.875$ ), was indeed obtained by means of the pr-EN and the WA (Equation 11b) methods, despite the differences in their basic assumptions. In this sense, the novel WA approach proved to be effective and of practical use especially when using Equation 11a, that is by considering the prestressing term $\sigma_{p}$ on the side of actions. The corresponding level of damage was in fact predicted in $D$ $=0.57$, that is with a marked reduction of the intrinsic error provided by the classical linear methods in use.

Due to the high scatter between the overall results obtained from the performed ULS verification comparisons, some further iterative calculations were also carried out by means of Equation 5 to 7 and 11a-b, by keeping fix all the input data for the design problem but changing the glass thickness $h$, Fig. 6 . 


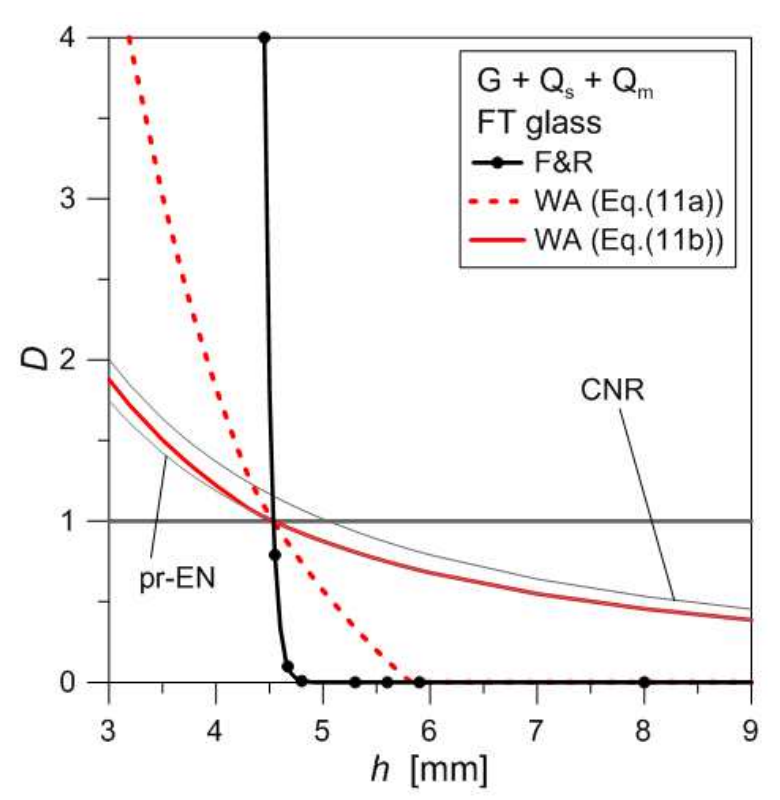

Fig. 6. Comparative analytical calculations for the FT laminated glass roof panel, as a function of the assigned thickness $h$

Compared to the AN worked example previously discussed, some differences were noticed, due to the presence of toughening treatments. A rather close correlation was found in terms of detection of the failure configuration (i.e., $D=1$ ) for all the examined methods. From Fig. 6, it can be seen in fact that for the given FT roof panel, an identical minimum glass thickness $h_{\text {min }}:=$ $D=1$ required to avoid collapse was obtained from the pr-EN, F\&R and WA approaches, while the CNR formulation still results conservative (but with a moderate margin of approximation, compared to the AN worked example).

The main difference of the so collected analytical estimations, as observed in the case of the AN example but further emphasized in Fig. 6 by the presence of the pre-stressing term $\sigma_{p}$, is given by the overall damage evolution in the FT element $(D<1)$, up to collapse. The pre-stressing contribution was in fact found to be typically so high-compared to the tensile stress ratio due to ordinary design actions of practical interest for structural glass elements that the analytical check required by some of the examined formulations would generally tend to simplify (see for example Equation 23 and 24).

The typically high sensitivity of the exponential F\&R formulation to the input geometrical and mechanical data, in this sense, further increases as far as the pre-stressing contribution increase. This finding can be also observed in Fig. 6, where compared to the AN glass panel of Fig. 5 the perception of lack of appropriate safety margins close to collapse further increases. This is not the case of the linear cumulative model proposed in the WA formulation, where the failure configuration is correctly detected and possible inconsistency in the design (i.e., minimum glass thickness, characteristic value of design actions, production tolerances, etc.) leading a given glass element close to collapse can be eventually perceived. As far as Equation 11a is taken into account i.e., with pre-stressing contributions on the side of actionsmoreover, rather interesting damage evolution estimations can be observed $(0 \leq D<1)$, compared to the exponential F\&R as well as to the linear pr-EN and CNR predictions.

\section{Parametric Analysis and General Discussion}

A final attempt of assessment for the examined prEN, CNR, F\&R and WA analytical formulations was carried out in the form of an extended parametric analytical investigation. In doing so, laminated panels composed of two glass layers with uniform thickness $h$ were considered, in accordance with Fig. 3.

Variations were then imposed in the form of magnitude and amplitude of variable loads $Q_{i}$ for the ULS combinations, overall dimensions of each laminated glass panel, type and thickness $h_{\text {int }}$ of the interlayer.

The so obtained parametric data with up to 400 different geometrical and loading configurations are proposed in Fig. 7 and divided by glass type (AN, HS and FT), in non-dimensional form. The ratio between the minimum glass thickness $h_{\min }$ required by the F\&R approach to prevent failure, i.e., $h_{\min }$ : $=D=1$, over the total span $L$ of each panel, is assumed as a reference parameter for the full set of examined cases. The so collected analytical values are hence compared in terms of $R_{h}$ ratio, being defined as:

$$
R_{h}=\frac{\left(h_{\min }\right)_{i}}{\left(h_{\min }\right)_{F \& R}}
$$

with $i$ denoting the pr-EN, CNR or WA methods respectively. Some interesting findings were observed from the extended parametric investigation, as also highlighted in Fig. 7.

Regarding the pr-EN formulation, in particular, it was noticed that:

- When no pre-stressing effects are considered for the glass elements to verify (Fig. 7a), due to the wrong assumptions of the method, markedly unsafe predictions were generally obtained for the detection of the failure configuration, with a margin of errors up to $30-40 \%$ the $F \& R$ exact solution 
- Despite the inconsistency of its basic assumptions, thanks to the beneficial pre-stressing contribution, the pr-EN formulation proved to offer a limited or almost null margin of error at failure for HS panels (Fig. 7b), compared to the F\&R exact formulation, with $\approx 0-2 \%$ (but still on the unsafe side) discrepancy

- The pr-EN formulation, finally, was found able to provide accurate detection of the failure configuration of a given structural glass member under a ULS combination of actions in the case of FT glass elements only (Fig. 7c). Based on the generally observed trends of the collected parametric results for $\mathrm{AN}, \mathrm{HS}$ and FT glass panels, as well as on the inconsistency of the assumptions supporting the method, however, the pr-EN approach confirmed to be not applicable for design purposes

Regarding the Palmgren-Miner based formulation in use in the CNR Italian document, the linear approach proved to be simple and practical in use. The same formulation, however, emphasized to be highly

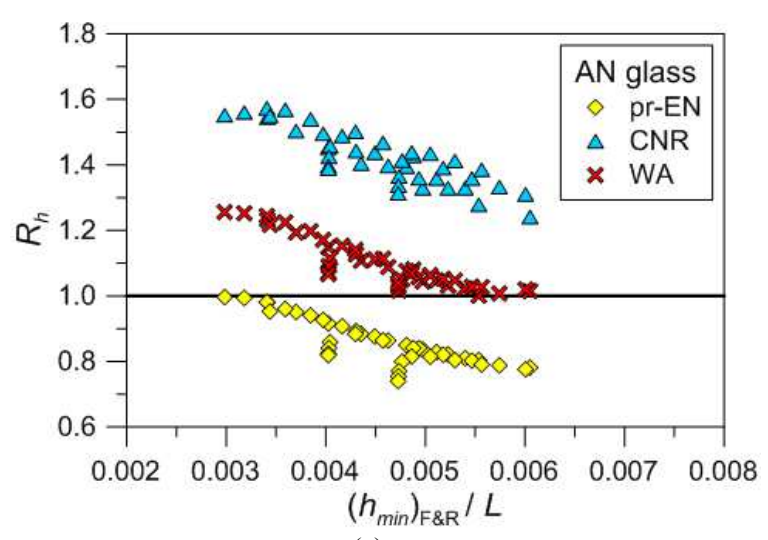

(a) conservative for the detection of the failure configuration in a given glass element, compared to the F\&R solution and in particular:

- A moderate scatter (always on the safe side) was generally observed in the case of pre-stressed glass elements. The margin of error from the exact F\&R solution was found to decrease with the amount of initial compressive stresses $\sigma_{p}$ and to lie in a range below $\approx 10 \%$ and $\approx 20 \%$ for FT glass and HS glass respectively

- For AN glass panels, large approximations were indeed noticed compared to the F\&R solutions, with average magnitude in the order of $\approx 25 \%$, but rising up also to $50-60 \%$, within specific loading configurations. In this sense, although still conservative, the CNR formulation could typically result in strong overestimation of the design of a given structural glass element, hence to not allow a combined structural optimization and safe-fail design approach

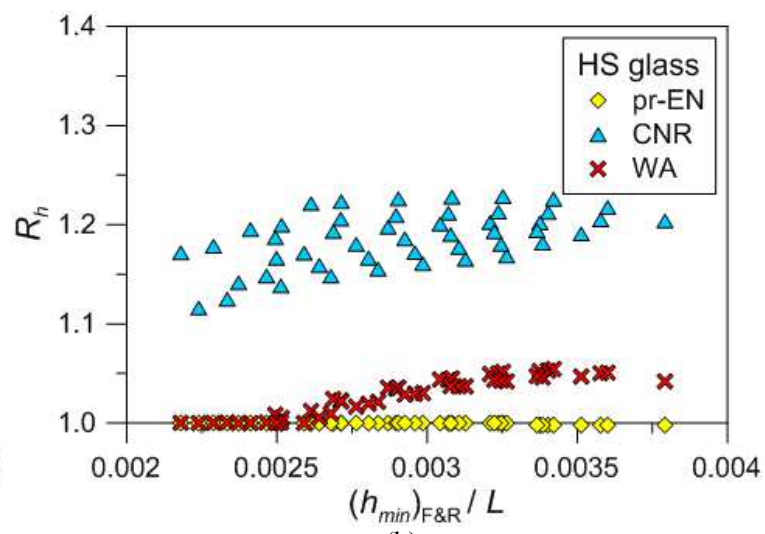

(b)

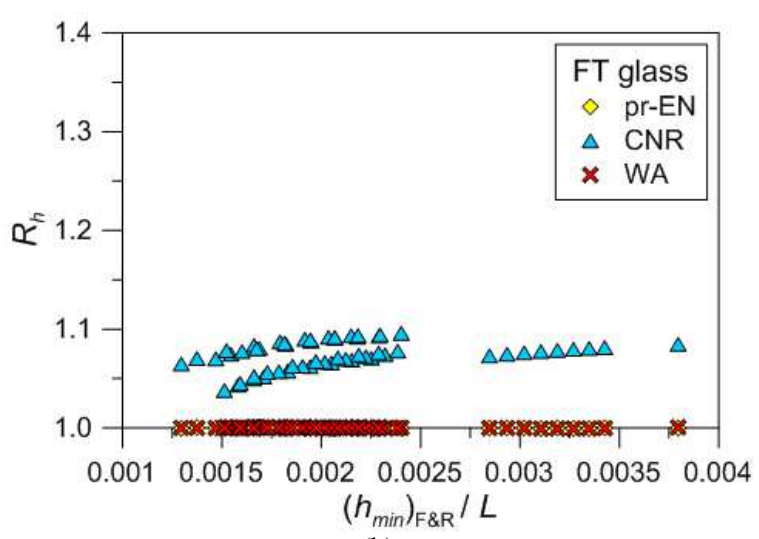

(b)

Fig. 7. Non-dimensional parametric comparisons for (a) AN, (b) HS and (c) FT glass panels under a ULS combination of variable loads 
In terms of the novel WA method proposed in this study, finally, an interesting correlation was typically observed through the extended parametric analyses. In particular:

- For FT glass panels, thanks to the strong prestressing effect on the total tensile stresses deriving from a general ULS combination of design loads, the WA solutions at failure $(D=1)$ were always found to coincide with the F\&R method

- In the case of HS glass panels, close correlation was also obtained at failure, with a margin of discrepancy (always on the safe side) from the F\&R method up to $\approx 5 \%$

- Largest approximations were indeed noticed for AN glass panels at failure $(D=1)$, compared to the $\mathrm{F} \& \mathrm{R}$ solutions. In any case, the average margin of error (always on the safe side) was found to be in the order of $\approx 5-10 \%$ for most of the loading configurations of practical interest for the design of structural glass elements

- As far as the WA method allows to account possible pre-stressing terms on the side of actions rather than on the side of the material design strength, a rather interesting prediction of failure evolution was generally observed (i.e., $D<1$ ), compared to the exponential F\&R formulation. As a result, based on the overall WA calculations and comparison, as well as on the simplicity and intuitivism of its linear formulation, it is expected that the WA approach could rationally represent a practical tool and a valid alternative to the F\&R non-linear method

Despite its robust background, the theoretically exact, non-linear F\&R approach-since formulated on the base of an exponential resisting domain-appeared in general highly sensitive to even small variations in the input parameters of the design problem (i.e., thickness of glass, magnitude of the assigned design loads, etc.). In this sense, a lack of appropriate safety margins was perceived when solving some typical design calculations. This perception would suggest (on the side of the professional engineer) a careful and rigorous design methodology, as well as (on the side of the committee in charge for the proposal of standardized design recommendations) the possible implementation of additional partial safety factors able to establish a more appropriate safety margin and to minimize the effect of possible errors in design.

\section{Summary and Conclusion}

In this study, an analytical investigation was dedicated to the ULS verification of structural glass elements under variably combined design actions.

An alternative, weighted average-based formulation (WA) was hence presented, taking advantage of existing formulations and literature proposals. Its advantage, as shown, is typically given by the simplicity and intuitivism of linear methods. At the same time, a key role is assigned to the weighted average calculation of an equivalent load duration coefficient $k_{\text {mod,w }}$ allowing to obtain accurate estimations for of a given glass element under a generic ULS combination of design actions.

The accuracy of the novel WA formulation was assessed towards three existing analytical formulations, including the linear cumulative approaches currently in use within the pr-EN European standard (prEN 16612: 2013, 2013) and the CNR Italian code (CNR-DT/2102013) for structural glass, as well as for the theoretically exact non-linear formulation proposed by Franco and Royer-Carfagni (2015, labelled in this study as 'F\&R') The latter exponential formulation, due to its robust theoretical background, was taken into account as a reference model for critical assessment of the novel WA approach, as well as of all the examined linear methods.

Based on an extended critical discussion of two worked example, as well as an extended series of parametric calculations of practical interest for designers, it was shown that the pr-EN approach, due to its wrong basic assumptions, can correctly detect the failure configuration in the case of FT glass panels only. As far as HS or AN glass panels are considered, the pr-EN method was found to lie always on the unsafe side, compared to the F\&R exact solution, with margins of errors up to $\approx 0-2$ and $30-40 \%$ respectively.

The CNR linear formulation also highlighted, despite the simplicity of the method, to be typically highly conservative. The scatter from the F\&R solution was found to decrease when increasing the pre-stressing beneficial contributions. For AN glass panels, however, this scatter was found to lie in the order of $\approx 25 \%$ and to increase up to $50-60 \%$ in certain loading configurations.

The novel WA formulation here proposed, finally, proved to represent a valid alternative to the exact exponential F\&R method. Thanks to the weighted average calculation of $k_{m o d, w}$ (inclusive of the stress ratio effect for each of the imposed design actions), the failure condition under a generic ULS combination of loads was generally predicted with a good level of accuracy, compared to the F\&R method. For FT panels, the solutions of the two methods were found to coincide. For HS and AN glass panels, the margin of scatter (always on the safe side) was found to lie below $5 \%$ and in the order of $10 \%$ respectively for all the examined configurations compared to the F\&R method.

\section{Author's Contributions}

Both the Authors actively contributed to the implementation of the proposed analytical model, as well as to the development of the parametric analyses and to the paper drafting, including a critical review of its content. 


\section{Ethics}

The article is original and contains unpublished material only. The corresponding author confirms that all the authors have read and approved the manuscript in its final form and no ethical issues are involved.

\section{References}

ASTM E1300-12ae1, 2002. Standard practice for determining load resistance of glass in buildings. The American Society for Testing and Materials.

Badalassi, M., L. Biolzi, G. Royer-Carfagni and W. Salvatore, 2014. Safety factors for the structural design of glass. Constr. Build. Mater., 55: 114-27.

Ballarini, R., G. Pisano and G. Royer-Carfagni, 2016. New calibration of partial material factors for the structural design of float glass. Comparison of bounded and unbounded statistics for glass strength. Constr. Build. Mater., 121: 69-80.

DOI: 10.1016/j.conbuildmat.2016.05.136

Beason, W. and L. Morgan, 1984. Glass failure prediction model. J. Struct. Eng., 110: 197-212. DOI: 10.1061/(ASCE)0733-9445(1984)110:2(197)

Bedon, C. and C. Amadio, 2015. Design buckling curves for glass columns and beams. Struct. Build., 168: 514-526. DOI: 10.1680/stbu.13.00113

Bedon, C. and C. Amadio, 2016. A unified approach for the shear buckling design of structural glass walls with non-ideal restraints. Am. J. Eng. Applied Sci., 9: 64-78. DOI: 10.3844/ajeassp.2016.64.78

Brown, W., 1972. A load duration theory for glass design. Publication no. NRC 12354, National Research Council of Canada, Division of Building Research.

CAN/CGSB 12.20-M89, 1989. Structural design of glass for buildings. Canadian General Standards Board.

CNR-DT 210/2013, 2013. Guide for the design, construction and control of structures made of glass structural elements (in Italian, Istruzioni per la Progettazione, l'Esecuzione ed il Controllo di Costruzioni con Elementi Strutturali di Vetro). National Research Council (CNR), Rome, Italy.

DIN 18008: 2010-12, 2010. Glass in building-design and construction rules. German Institute for Standardisation (Deutsches Institut für Normung).

EN 1990: 2002, 2002. Basis of structural design. European Standard, European Committee for Standardization (CEN), Brussels, Belgium.

Feldmann, M., R. Kasper, B. Abeln, P. Cruz and J. Belis et al., 2014. Guidance for European structural design of glass components-support to the implementation, harmonization and further development of the Eurocodes. Report EUR 26439-Joint Research Centre-Institute for the Protection and Security of the Citizen. Eds. Dimova, Pinto, Feldmann, Denton.
Fischer-Cripps, A. and R. Collins, 1995. Architectural glazings: Design standards and failure models. Build Environ., 30: 29-40.

DOI: 10.1016/0360-1323(94)E0026-N

Franco, A. and G. Royer-Carfagni, 2015. Verification formulae for structural glass under combined variable loads. Eng. Struct., 83: 233-242.

DOI: $10.1016 /$ j.engstruct.2014.10.049

Galuppi, L. and G. Royer-Carfagni, 2012. Effective thickness of laminated glass beams: New expression via a variational approach. Eng. Struct., 38: 53-67. DOI: 10.1016/j.engstruct.2011.12.039

Hagl, A., 2016. Development and test logics for structural silicone bonding design and sizing. Glass Struct. Eng., 1 131-151. DOI: $10.1007 / \mathrm{s} 40940-016-0014-5$

Larcher, M., M. Arrigoni, C. Bedon, A. van Doormaal and C. Haberacker et al., 2016. Design of blastloaded glazing windows and facades: A review of essential requirements towards standardization. Adv. Civil Eng., 2016: 2604232-2604232. DOI: $10.1155 / 2016 / 2604232$

Maniatis, I. and M. Elstner, 2016. Investigations on the mechanical strength of enamelled glass. Glass Struct. Eng., 1: 277-288. DOI: $10.1007 / \mathrm{s} 40940-016-0025-2$

NEN 2608: 2011, 2011. Glass in building-requirements and determination method. Nederlands Normalisatie Instituut.

Overend, M., 2010. Recent developments in design methods for glass structures. Struct Eng., 88: 18-26.

ÖNORM B 3716, 2013. Glass in building-Structural glass construction. Osterreichisches Normungsinstitut

prEN 13474-1: 2007, 2007. Glass in building-Design of glass panes-Part 1: General basis of design. European Committee for Standardization (CEN), Brussels, Belgium.

prEN 16612: 2013, 2013. CEN/TC 250. Glass in building-Determination of the load resistance of glass panes by calculation and testing. European Committee for Standardization (CEN), Brussels, Belgium.

Sedlacek, G., K. Blank and J. Gusgen, 1995. Glass in structural engineering. Struct. Eng., 73: 17-22.

Wiederhorn, S. and L. Bolz, 1970. Stress corrosion and static fatigue of glass. J. Am. Ceram Soc., 53: 543-548.

DOI: $10.1111 /$ j.1151-2916.1970.tb15962.x 